\title{
Evaluation de l'exposition de la population aux métaux traces (cadmium, mercure, plomb) à travers la consommation des viandes et abats de bouf et de porc importés
}

\author{
Mathias Kouamé KOFFI ${ }^{1,2^{*}}$, Yolande AKE ASSI ${ }^{2}$, Justin S. SAKI ${ }^{3}$ et \\ Henri Marius G. A. BIEGO ${ }^{1}$ \\ ${ }^{1}$ Laboratoire de Biochimie et Sciences des Aliments (LaBSA), UFR Biosciences, \\ Université de Cocody, Abidjan, 22 BP 582 Abidjan 22, Côte d'Ivoire. \\ ${ }^{2}$ Laboratoire Central pour l'Hygiène Alimentaire et l'Agroindustrie (LCHAI), \\ Ministère de l'Agriculture, Côte d'Ivoire. \\ ${ }^{3}$ Laboratoire de Biotechnologie et Sciences des Aliments, UFR Biosciences, \\ Université Félix Houphouet Boigny de Cocody-Abidjan, 22 BP 582 Abidjan 22, Côte d'Ivoire. \\ *Auteur correspondant, E-mail : djetoplus@yahoo.fr, Tel. : (+225) 07854371
}

\section{RESUME}

Cette étude a pour objectif d'évaluer le niveau de contamination en éléments traces métalliques (ETM) toxiques $(\mathrm{Cd}, \mathrm{Hg}, \mathrm{Pb})$ dans les viandes et abats importés en vue de calculer l'exposition à long terme de la population ivoirienne. Pour ce faire, 192 échantillons prélevés sur la viande et les abats importés ont été analysés par spectrophotométrie d'absorption atomique. Il ressort que les traces des différents métaux existent dans les matrices à des taux variables avec un dépassement des critères de comestibilité au niveau du plomb. Le rognon avec $66,32 \mu \mathrm{g} / \mathrm{kg}$ de $\mathrm{Cd}, 21,00 \mu \mathrm{g} / \mathrm{kg}$ de $\mathrm{Hg}$ et 235,60 $\mu \mathrm{g} / \mathrm{kg}$ de $\mathrm{Pb}$, est l'organe le plus contaminé.

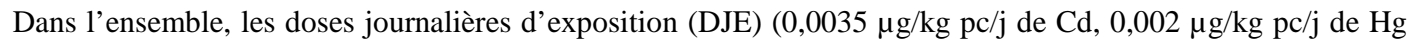
et $0,017 \mu \mathrm{g} / \mathrm{kg} \mathrm{pc} / \mathrm{j} \mathrm{de} \mathrm{Pb}$ ) sont inférieures aux doses journalières tolérées (DJT) respectives. Aussi le risque peut-il être écarté pour la population générale. Les traces métalliques existent dans les viandes et abats importés. Si les niveaux restent dans l'ensemble inférieurs aux seuils limites, dans le rognon, les teneurs en plomb sont élevées, augmentant le risque d'intoxication. En effet, il suffit de consommer quotidiennement 100 g de rognon pour que le risque soit à plus de $150 \%$ de la DJT au niveau de certains éléments métalliques.

(C) 2014 International Formulae Group. All rights reserved.

Mots clés : Viande, abats, contamination métallique, exposition, Côte d'Ivoire.

\section{INTRODUCTION}

Les enjeux soulevés par les émissions des éléments métalliques traces (EMT) sont principalement sanitaires et sont liés à leur persistance dans le milieu naturel, à leur caractère bio-accumulateur dans l'environnement et à leurs effets sur la santé (SETRA, 2004). Un grand nombre de contaminants d'origine et de nature aussi variées que diverses peuvent contaminer les sols, l'environnement et être captés par la chaîne alimentaire. Une fois transférés à l'être humain par la voie digestive, ils se combinent aux composés organiques soufrés de notre organisme pour engendrer de graves troubles, y compris au niveau cérébral (Picot, 2003), à 
court ou à long terme. En l'absence de toute source spécifique d'exposition, l'alimentation constitue la voie principale d'exposition à nombre de ces métaux. Aussi, les crises alimentaires induites par la pollution environnementale ont généré la revendication de la part des consommateurs, un droit à la sécurité alimentaire (Claire et al., 2005). D'où la fixation par les organismes compétents de normes et de valeurs limites quantitatives de sécurité (valeurs toxicologiques de référence) au delà desquelles la santé serait menacée. Ainsi, évaluer le niveau de contamination de ces métaux dans les organismes terrestres permet-elle de rendre compte de la qualité chimique du milieu et des aliments. Lorsque ces éléments existent dans l'alimentation des ruminants (bétail), ils sont accumulés dans les reins et foies de ces animaux (EFSA, 2009 ; 2010). Ces organes représentent de ce fait, une source non négligeable d'apport exogène d'origine alimentaire (Boumehres, 2010) de ces métaux, dès lors qu'ils sont consommés par les humains.

En Côte d'Ivoire, du fait d'une non autosuffisance en produits carnés, des importations massives de ces produits sont faites sous forme d'animaux vivants (bovins, caprins, ovins et volailles) des pays limitrophes et sous forme congelée des pays lointains à travers rognons, foies, sternum, viandes, côtelettes, cœur, etc. Certaines de ces denrées sont des sources potentielles de contamination alimentaire par des toxiques et pour qui des limites ont été fixées par la législation. Cette étude se propose de déterminer le niveau de contamination des viandes et abats importés par les toxiques métalliques tels que le cadmium, le mercure et le plomb, et évaluer l'exposition alimentaire à ces toxiques de la population générale.

\section{MATERIEL ET METHODES \\ Echantillonnage}

L'échantillonnage qui s'est déroulé sur douze (12) mois, a été effectué par les services officiels d'inspection vétérinaire au port chez des importateurs agréés. Ainsi, les échantillons de foie, de rognon, de sternum et de viande sont acheminés au laboratoire en unité d'emballage. Tous les cartons de même nature de la semaine constituent l'échantillon. Les analyses des métaux ont été réalisées au Laboratoire Central pour l'Hygiène Alimentaire et l'Agro-Industrie (LCHAI), sur des échantillons composites obtenus à partir des parties comestibles de plusieurs spécimens. Après dégivrage, les aliquotes de $50 \mathrm{~g}$ sont prélevées sur les cartons, broyées et mélangées pour former l'échantillon de la semaine. Au total, 192 échantillons ont été analysés dont 48 par matrice.

\section{Traitement des échantillons et analyse}

Les analyses des métaux ont été réalisées selon des modes opératoires parfaitement maîtrisés et validés. La minéralisation pour le dosage du plomb et du cadmium a été effectuée selon la méthode AOAC, 999.10 (2003). A partir d'un homogénat de chair, les fractions de $500 \mathrm{mg}$ ont été prélevées et attaquées par $5 \mathrm{ml}$ d'acide nitrique $(65 \% \mathrm{w} / \mathrm{w})$ et $2 \mathrm{ml}$ d'eau oxygénée $30 \%$ au four à micro-onde, type ETHOS Milestone à $200{ }^{\circ} \mathrm{C}$ pendant 45 minutes. Après cette attaque, le minéralisât a été récupéré dans une fiole de $25 \mathrm{ml}$ et complété au trait de jauge avec de l'eau bi distillée. Les mesures ont été effectuées grâce à un spectrophotomètre d'absorption atomique de marque VARIAN, type SPECTRA AA110 équipé d'un four graphite GTA 110 et la lampe de Deutérium pour correction du bruit de fond.

Pour le mercure, la méthode AOAC, 971.21 (2000) a été appliquée. Sur la minéralisation effectuée pour le cadmium et le plomb, une prise d'essai de $10 \mathrm{ml}$ a été faite. Après bromisation, le dosage a été effectué par fluorescence atomique après formation de vapeurs froides en présence de chlorure stanneux. Le même SPECTRA 
AA110 a été utilisé cette fois en mode vapeur froide grâce à un générateur d'hydrure (VGA 77). La lampe de Deutérium a été utilisée pour correction du bruit de fond.

Les différentes déterminations ont été faites à l'aide de droites d'étalonnage établies en cinq (5) points. Les prises d'essais ont été faites en triple. Pour chaque série d'analyse, un blanc de digestion et un échantillon de référence de concentration connue ont été analysés pour assurer le contrôle qualité des résultats.

La totalité de chaque élément a été mesurée par ces méthodes, indépendamment de leur forme chimique. L'évaluation de l'exposition aux différents métaux de la population nécessite l'exploitation de deux sources de données :

- les données relatives aux quantités d'abats consommées par la population,

- les résultats des mesures des éléments métalliques dans les abats.

Nous adoptons une approche déterministe en croisant les données moyennes de consommation et de concentration pour calculer une dose journalière d'exposition aux trois métaux traces métalliques via la consommation des abats. $\mathbf{A}=\mathbf{C i} \times \mathbf{Q}$, où $A$ est l'apport en élément métallique ; $\mathrm{Ci}$ est la concentration en $\mu \mathrm{g} / \mathrm{kg}$ de l'élément toxique et $\mathrm{Q}$ représente la quantité de consommation d'abats. Cette valeur est la moyenne nationale obtenue auprès des services compétents.

Dans cette étude, nous émettons des hypothèses suivantes :

- La consommation par la voie alimentaire est la seule source prise en compte

- La consommation de viande et d'abats est régulière et journalière

- La consommation est identique pour les enfants et les adultes.

- L'exposition prise en compte ici est l'exposition orale due à la consommation des viandes et abats importés.
- Le mercure total dosé est considéré à 100\% comme le méthylmercure.

\section{Expression des résultats}

Les résultats sont exprimés en microgramme par kilogramme ( $\mu \mathrm{g} / \mathrm{kg}$ ou $\mathrm{ppb})$ de poids frais. Les valeurs moyennes sont affectées de l'écart-type $(m \pm$ EC) et flanquées des valeurs extrêmes de minima et maxima. La variabilité des teneurs métalliques est étudiée par une analyse de variance à un facteur effectuée par la procédure ANOVA du logiciel STATISTICA 7.1. Les comparaisons des teneures moyennes au niveau des organes ont été réalisées par le test de TUKEY chaque fois que l'analyse de variance révèle des différences significatives. Par rapport à l'exposition, après analyse, les valeurs inférieures au seuil de quantification ont été enregistrées comme égales à ce seuil afin de surestimer cette exposition.

\section{RESULTATS}

\section{Contamination}

Les résultats consignés dans le Tableau 1 indiquent clairement que les traces des métaux étudiés existent dans les différentes matrices et ce à divers niveaux. Les données montrent une différence significative entre les matrices et les toxiques. Ainsi, le rognon est la matrice la plus contaminée quand le plomb reste le métal le plus accumulé.

Le cadmium a été détecté dans $92,70 \%$ des échantillons. Sa répartition est inégale dans les différentes matrices. Sa concentration rénale est significativement différente des autres. Ses traces varient entre $0,37 \mu \mathrm{g} / \mathrm{kg}$ et $301 \mu \mathrm{g} / \mathrm{kg}$ et une valeur moyenne de 28,40 $\mu \mathrm{g} / \mathrm{kg}$.

Le mercure est présent dans $91,15 \%$ des échantillons où sa concentration varie peu entre $3,4 \mu \mathrm{g} / \mathrm{kg}$ et $69 \mu \mathrm{g} / \mathrm{kg}$ pour une valeur moyenne de $16,61 \mu \mathrm{g} / \mathrm{kg}$. 
Le plomb est le toxique le plus concentré d'eux tous. Détecté dans 91,67\% des échantillons, il cumule en moyenne à $140,36 \mu \mathrm{g} / \mathrm{kg}$ avec un pic de 235,60 $\mu \mathrm{g} / \mathrm{kg}$ dans le rognon. Il existe une différence significative au niveau de sa répartition matricielle.

Dans le foie, le mercure et le plomb ont été détectés sur 42 échantillons $(87,5 \%)$ contre $46(95,83 \%)$ pour le cadmium. Les teneurs moyennes sont: $16,61 \mu \mathrm{g} / \mathrm{kg} ; 90,60$ $\mu \mathrm{g} / \mathrm{kg}$ et $19,80 \mu \mathrm{g} / \mathrm{kg}$ de poids frais respectivement. Les valeurs détectées du mercure varient entre $3,4 \mu \mathrm{g} / \mathrm{kg}$ et $57 \mu \mathrm{g} / \mathrm{kg}$. Les concentrations du plomb varient entre 6,5 $\mu \mathrm{g} / \mathrm{kg}$ et $189 \mu \mathrm{g} / \mathrm{kg}$ et celle du cadmium sont comprises entre $0,3 \mu \mathrm{g} / \mathrm{kg}$ et $65 \mu \mathrm{g} / \mathrm{kg}$.

Le rognon est selon nos résultats l'organe qui accumule le plus les différents métaux. Dans celui-ci, le mercure est présent dans tous les échantillons où les teneurs varient de $5,7 \mu \mathrm{g} / \mathrm{kg}$ à $67 \mu \mathrm{g} / \mathrm{kg}$ pour une valeur moyenne de $21 \mu \mathrm{g} / \mathrm{kg}$.

Le plomb détecté seulement sur 43 échantillons soit $89,59 \%$ cumule entre 6,5 $\mu \mathrm{g} / \mathrm{kg}$ et $1597 \mu \mathrm{g} / \mathrm{kg}$ avec une valeur moyenne $235,60 \mu \mathrm{g} / \mathrm{kg}$

Le cadmium rénal a été détecté dans 46 échantillons $(95,83 \%$ des échantillons de rein analysés). La valeur maximale quantifiée est de $301 \mu \mathrm{g} / \mathrm{kg}$ de poids frais. La valeur moyenne est de $66.32 \mu \mathrm{g} / \mathrm{kg}$.

Pour le sternum, le mercure n'a pas été détecté dans cinq (5) échantillons. Pour les autres, les taux varient entre $3,4 \mu \mathrm{g} / \mathrm{kg}$ et 48 $\mu \mathrm{g} / \mathrm{kg}$ avec une valeur moyenne de $13,91 \mu \mathrm{g} / \mathrm{kg}$.

Avec une valeur moyenne de 111,40 $\mu \mathrm{g} / \mathrm{kg}$ le plomb du sternum varie entre 6,5 $\mu \mathrm{g} / \mathrm{kg}$ et $254 \mu \mathrm{g} / \mathrm{kg}$ retrouvé dans 46 échantillons. Quant au cadmium, sa concentration moyenne est de $12,81 \mu \mathrm{g} / \mathrm{kg}$ et elle varie entre $0,37 \mu \mathrm{g} / \mathrm{kg}$ et $31 \mu \mathrm{g} / \mathrm{kg}$ qu'on retrouve dans 43 échantillons sur 48 analysés.

En dehors de 2 échantillons, tous les autres échantillons de muscle (Viande) contiennent les traces de mercure. Ces traces varient entre $3,4 \mu \mathrm{g} / \mathrm{kg}$ et $69 \mu \mathrm{g} / \mathrm{kg}$ de poids frais avec une valeur moyenne de 14,94 $\mu \mathrm{g} / \mathrm{kg}$.

Avec une valeur moyenne de 123,90 $\mu \mathrm{g} / \mathrm{kg}$ pour le plomb et $14,65 \mu \mathrm{g} / \mathrm{kg}$ pour le cadmium, ces deux métaux ont été détectés respectivement dans 45 et 44 échantillons de muscle (viande) et leurs traces varient entre $6,5 \mu \mathrm{g} / \mathrm{kg}$ et $281 \mu \mathrm{g} / \mathrm{kg}$ pour le plomb et 0,37 $\mu \mathrm{g} / \mathrm{kg}$ et $43 \mu \mathrm{g} / \mathrm{kg}$ pour le cadmium.

\section{Exposition}

L'exposition calculée par croisement de la consommation et des valeurs moyennes des traces métalliques est consignée dans le Tableau 2.

A partir de l'absorption journalière de $0,21 \mu \mathrm{g}$ de cadmium, on déduit une exposition journalière au cadmium de $0.0035 \mu \mathrm{g} / \mathrm{kg} \mathrm{pc} / \mathrm{j}$ pour l'adulte de $60 \mathrm{~kg}$ et $0.01 \mu \mathrm{g} / \mathrm{kg} \mathrm{pc} / \mathrm{j}$ pour le jeune de $20 \mathrm{~kg}$ (soit environ 3 fois la charge des adultes).

Le mercure ingéré quotidiennement à travers la consommation des viandes et abats de porc et bœuf est de $0,12 \mu \mathrm{g}$, soit une dose journalière d'exposition (DJE) de $0.002 \mu \mathrm{g} / \mathrm{kg}$ $\mathrm{pc} / \mathrm{j}$.

La quantité de plomb à laquelle s'expose le consommateur adulte de viande et abats de bœuf et porc importés est de $1.05 \mu \mathrm{g}$, soit une dose journalière d'exposition de $0.017 \mu \mathrm{g} / \mathrm{kg} \mathrm{pc} / \mathrm{j}$. Le jeune adolescent de moins de 14 ans $(20 \mathrm{~kg})$ s'expose à 0,053 $\mu \mathrm{g} / \mathrm{kg} \mathrm{pc} / \mathrm{j}$ soit 3 fois plus que l'adulte. 
Tableau 1 : Concentration en éléments toxiques des abats importés en Côte d'Ivoire en $2010(\mu \mathrm{g} / \mathrm{kg}$ de poids frais).

\begin{tabular}{lcccc}
\hline Organes & Effectif & Cd & Hg & Pb \\
\hline \multirow{2}{*}{ Foie } & \multirow{2}{*}{48} & $\mathbf{1 9 . 8 0} \pm \mathbf{1 5 . 7 0}$ & $\mathbf{1 6 . 6 1} \pm \mathbf{1 4 . 8 7}$ & $\mathbf{9 0 . 6 0} \pm \mathbf{5 5 . 5 5}$ \\
& & $(0.37-65)$ & $(3.4-57)$ & $(6.5-189)$ \\
\hline \multirow{2}{*}{ Rognon } & \multirow{2}{*}{48} & $\mathbf{6 6 . 3 2} \pm \mathbf{7 3 . 3 1}$ & $\mathbf{2 1 . 0 0} \pm \mathbf{1 4 . 1 3}$ & $\mathbf{2 3 5 . 6 0} \pm \mathbf{3 3 1 . 9 3}$ \\
& & $(0.37-301)$ & $(5.7-67)$ & $(6.5-1597)$ \\
\hline \multirow{2}{*}{ Sternum } & \multirow{2}{*}{48} & $\mathbf{1 2 . 8 1} \pm \mathbf{7 . 7 5}$ & $\mathbf{1 3 . 9 1} \pm \mathbf{8 . 0 0}$ & $\mathbf{1 1 1 . 4 0} \pm \mathbf{6 4 . 6 0}$ \\
& \multirow{2}{*}{ Viande } & $(0.37-31)$ & $(3.4-48)$ & $(6.5-254)$ \\
\hline \multirow{2}{*}{ Ensemble } & \multirow{2}{*}{19} & $\mathbf{1 4 . 6 5} \pm \mathbf{1 0 . 1 4}$ & $\mathbf{1 4 . 9 4} \pm \mathbf{1 0} \mathbf{. 8 5}$ & $\mathbf{1 2 3 . 9 0} \pm \mathbf{5 4 . 4 0}$ \\
& \multirow{2}{*}{192} & $\mathbf{2 8 . 4 0} \pm \mathbf{4 3 . 8 7}$ & $\mathbf{1 6 . 6 1} \pm \mathbf{1 2 . 5 4}$ & $\mathbf{1 4 0 . 3 6} \pm \mathbf{1 8 1 . 9 6}$ \\
& & $(0.37-301)$ & $(3.4-69)$ & $(6.5-1597)$ \\
\hline
\end{tabular}

Tableau 2 : Apports journaliers en éléments métalliques des viandes et abats de bœuf et porc importés.

\begin{tabular}{|c|c|c|c|c|}
\hline Elément & & Cadmium & Mercure & Plomb \\
\hline Ingestion $(\mu \mathrm{g} / \mathrm{j})$ & & 0,21 & 0,12 & 1,06 \\
\hline \multirow{2}{*}{ DJE $(\mu \mathrm{g} / \mathrm{kg} \mathrm{pc/j})$} & Adulte de $60 \mathrm{~kg}$ & 0.0035 & 0.002 & 0.017 \\
\hline & Enfant de $20 \mathrm{~kg}$ & 0.01 & 0.006 & 0.053 \\
\hline DJT $(\mu \mathrm{g} / \mathrm{kg} \mathrm{pc/j})$ & & 1 & 0.23 & 3.6 \\
\hline \multirow{2}{*}{ DJE/DJT } & Adulte de $60 \mathrm{~kg}$ & 0.0035 & 0.0087 & 0.0047 \\
\hline & Enfant de $20 \mathrm{~kg}$ & 0.01 & 0.026 & 0.01 \\
\hline
\end{tabular}

Tableau 3: Métaux lourds toxiques dans l'alimentation en France (Source : CSHPF, 1996).

\begin{tabular}{lcc}
\hline Mercure & Plomb & Cadmium \\
\hline Poissons 35\% & Boisson 33\% & Fruits et légumes 30\% \\
Coquillage 6\% & Fruits et légumes 22\% & Produits laitiers 19\% \\
Crustacés 3.4\% & Viande poisson abats & Céréales 16\% \\
Fruits et légumes $14 \%$ & $16 \%$ Produits laitiers & Abats 6\% \\
champignons $0.2 \%$ & $14 \%$ Céréales 13\% & Poissons 2.6\% \\
Viandes et abats $9.3 \%$ & & Charcuterie 3\% \\
\hline
\end{tabular}

\section{DISCUSSION}

La réglementation a fixé en tenant compte de plusieurs paramètres d'une part, les teneurs maximales pour certains contaminants dont le cadmium, le mercure et le plomb, dans les denrées alimentaires et d'autres part les doses périodiques (journalière ou hebdomadaire) à ingérer sans risque d'intoxication alimentaire pour les populations.

$\mathrm{Si}$ les produits carnés ne sont pas considérés comme source alimentaire potentielle du mercure, il n'en est pas de même pour le plomb et le cadmium pour 
lesquels le règlement (CE : 1881/2006) fixe à $50 \mu \mathrm{g} / \mathrm{kg}, 500 \mu \mathrm{g} / \mathrm{kg}, 1000 \mu \mathrm{g} / \mathrm{kg}$ les teneurs maximales du cadmium dans les viandes (bovine et ovine), dans le foie et dans le rognon respectivement et à $100 \mu \mathrm{g} / \mathrm{kg}$ de plomb dans la viande (bovin et ovin) et à $500 \mu \mathrm{g} / \mathrm{kg}$ dans les abats.

$\mathrm{Au}$ vu des textes en vigueur, on déduit que nos valeurs du cadmium sont inférieures aux valeurs de référence tant globalement que spécifiquement. En effet, la valeur moyenne du cadmium dans l'ensemble des échantillons $(28,40 \mu \mathrm{g} / \mathrm{kg})$ est inférieure à $50 \mu \mathrm{g} / \mathrm{kg}$ (valeur minimale), aussi, les valeurs moyennes de la viande $(14,65 \mu \mathrm{g} / \mathrm{kg})$, du foie $(19,80 \mu \mathrm{g} / \mathrm{kg})$ et du rognon $(66,32 \mu \mathrm{g} / \mathrm{kg})$ sont elles aussi inférieures aux valeurs guides de ces matrices. Dans la littérature, certaines données ne concordent pas avec les nôtres. Les études de Leblanc et al. (2004, 2011) donnent comme valeur cadmique de la viande $1 \mu \mathrm{g} / \mathrm{kg}$ et des abats $51,6 \mu \mathrm{g} / \mathrm{kg}$ et $1,2 \mu \mathrm{g} / \mathrm{kg}$ pour la viande contre $52,6 \mu \mathrm{g} / \mathrm{kg}$ pour les abats respectivement. L'exposition au cadmium $(0,21 \mu \mathrm{g} /$ per $/ \mathrm{j}$ ou $0,0035 \mu \mathrm{g} / \mathrm{kg} \mathrm{pc} / \mathrm{j})$ qui en découle est non seulement inférieure à la dose journalière tolérable fixée par l'OMS qui est de $1 \mu \mathrm{g} / \mathrm{kg} \mathrm{pc} / \mathrm{j}$ (OMS, 2006), mais, est aussi inférieure aux valeurs rencontrées dans la littérature et qui prennent en compte l'ensemble de l'alimentation. En effet, les différentes études françaises rapportent une exposition de $23 \mu \mathrm{g} / \mathrm{per} / \mathrm{j}$ soit $0,38 \mu \mathrm{g} / \mathrm{kg} \mathrm{pc} / \mathrm{j}$ (DGS, 1992), 19,6 $\mu \mathrm{g} / \mathrm{per} / \mathrm{j}$ soit une dose de 0,33 $\mu \mathrm{g} / \mathrm{kg}$ pc/j (Décloitre, 1998), $36 \mu \mathrm{g} / \mathrm{per} / \mathrm{j}$ pour $0,60 \mu \mathrm{g} / \mathrm{kg} \mathrm{pc} / \mathrm{j}$ (Noel et al., 2003) pour les adultes de $60 \mathrm{~kg}$. Pour une population de plus 15 ans, l'exposition au cadmium s'élève à $2,7 \mu \mathrm{g} / \mathrm{per} / \mathrm{j}$ et à $2 \mu \mathrm{g} / \mathrm{per} / \mathrm{j}$ pour les plus jeunes (Leblanc, 2004). L'étude de l'alimentation totale de Leblanc et al. (2011) donne la contribution de la viande et des abats à l'exposition qui était de $9,6 \mu \mathrm{g} / \mathrm{per} / \mathrm{j}$ soit $0,16 \mu \mathrm{g} / \mathrm{kg}$ pc/j pour les adultes à $4,8 \mu \mathrm{g} / \mathrm{per} / \mathrm{j}$ ou $0,24 \mu \mathrm{g} / \mathrm{kg}$ pc/j pour les jeunes enfants. La contribution des viandes et abats étant de $0,009 \mu \mathrm{g} / \mathrm{kg} \mathrm{pc} / \mathrm{j}$, reste nettement plus élevée que notre valeur d'exposition au cadmium évaluée à $0,0035 \mu \mathrm{g} / \mathrm{kg} \mathrm{pc} / \mathrm{j}$. Au niveau du plomb, nos résultats sont supérieurs aux valeurs toxicologiques de référence de la viande et du sternum $(100 \mu \mathrm{g} / \mathrm{kg})$ qui ont respectivement des teneurs moyennes de $123,90 \mu \mathrm{g} / \mathrm{kg}$ et de $111,40 \mu \mathrm{g} / \mathrm{kg}$. Les données relatives aux abats sont cependant inférieures avec $90,60 \mu \mathrm{g} / \mathrm{kg}$ pour le foie et $235,60 \mu \mathrm{g} / \mathrm{kg}$ pour le rognon contre $500 \mu \mathrm{g} / \mathrm{kg}$ fixé par les textes en vigueur. Par rapport au plomb, $11,45 \%$ des abats et $41,66 \%$ de la viande ont des concentrations supérieures à leurs valeurs guides respectives $(100 \mu \mathrm{g} / \mathrm{kg}$ et $500 \mu \mathrm{g} / \mathrm{kg}$ ). Nos valeurs sont aussi supérieures aux données en France où les teneurs moyennes du plomb rencontrées dans la viande et les abats sont de $4 \mu \mathrm{g} / \mathrm{kg}$ et 55 $\mu \mathrm{g} / \mathrm{kg}$ respectivement (Leblanc et al., 2004) et de $8 \mu \mathrm{g} / \mathrm{kg}$ pour la viande et $20 \mu \mathrm{g} / \mathrm{kg}$ pour les abats (Leblanc, 2011).

A partir de ces données, la quantité de plomb absorbée au quotidien par le consommateur ivoirien est de $1,06 \mu \mathrm{g}$ de laquelle découle une dose journalière d'exposition de $0,017 \mu \mathrm{g} / \mathrm{kg} \mathrm{pc} / \mathrm{j}$ pour l'adulte de $60 \mathrm{~kg}$ et de $0,053 \mu \mathrm{g} / \mathrm{kg} \mathrm{pc} / \mathrm{j}$ pour le jeune adolescent de $20 \mathrm{~kg}$ (soit trois fois la dose de l'adulte). Par rapport à la dose journalière tolérable fixée à $3,6 \mu \mathrm{g} / \mathrm{kg} \mathrm{pc} / \mathrm{j}$ par l'OMS (2006), nous déduisons que la contribution des viandes et abats importés à l'exposition au plomb par l'alimentation reste infime. Cette exposition est aussi inférieure à l'exposition en France estimée à $73 \mu \mathrm{g} / \mathrm{kg} \mathrm{pc} / \mathrm{j}$ pour les enfants et $123 \mu \mathrm{g} / \mathrm{kg} \mathrm{pc} / \mathrm{j}$ pour les adultes (DGS, 1992), ou à $68 \mu \mathrm{g} / \mathrm{kg}$ pc/j (Decloître ,1998), à $52 \mu \mathrm{g} / \mathrm{kg}$ pc/j (Leblanc et al., 2000), à $31 \mu \mathrm{g} / \mathrm{kg} \mathrm{pc} / \mathrm{j}$ (Noel et al., 2003), à $18 \mu \mathrm{g} / \mathrm{kg}$ $\mathrm{pc} / \mathrm{j}$ pour les adultes de plus de 15 ans et à 13 $\mu \mathrm{g} / \mathrm{kg} \mathrm{pc} / \mathrm{j}$ pour les adolescents dont l'âge est compris entre 3 et 14 ans (Leblanc et al., 2011).

En dehors du rognon $(21 \mu \mathrm{g} / \mathrm{kg})$, les concentrations du mercure dans les autres matrices varient peu et gravitent toutes autour de la valeur moyenne $16,61 \mu \mathrm{g} / \mathrm{kg}$. Les produits carnés n'étant pas des sources d'intoxication, il n'existe donc pas de valeur guide. Dans la littérature, les données de Leblanc et al. (2004, 2011) restent comparables aux nôtres. En effet, lors de ces deux études, il a évalué le mercure à $3 \mu \mathrm{g} / \mathrm{kg}$ et à $19 \mu \mathrm{g} / \mathrm{kg}$ respectivement dans la viande et dans les abats en 2004 contre $5 \mu \mathrm{g} / \mathrm{kg}$ dans les deux types de produit en 2011. La contribution des viandes et abats à 
l'exposition alimentaire mercurielle est négligeable. Notre étude révèle qu'elle s'évalue à $0,002 \mu \mathrm{g} / \mathrm{kg} \mathrm{pc} / \mathrm{j}$ pour l'adulte contre $0,23 \mu \mathrm{g} / \mathrm{kg} \mathrm{pc} / \mathrm{j}$ déduite de la DHTP fixée par l'OMS (2003) soit environ $0,8 \%$ Ailleurs, les données d'exposition au mercure sont disparates. Pour la DGS (1992) elles étaient de $0,6 \mu \mathrm{g} / \mathrm{kg} \mathrm{pc} / \mathrm{j}$ pour les enfants et $0,26 \mu \mathrm{g} / \mathrm{kg} \mathrm{pc} / \mathrm{j}$ pour les adultes. Decloître (1998) a évalué à $15 \mu \mathrm{g} / \mathrm{j}$ dans l'ensemble quant Leblanc et al. (2004) indiquaient 9,7 $\mu \mathrm{g} / \mathrm{j}$ pour les adultes et $7,9 \mu \mathrm{g} / \mathrm{j}$ pour les adolescents de 3 à 14 ans. Les derniers travaux de Leblanc (2011) donnent 0,017 $\mu \mathrm{g} / \mathrm{kg} \mathrm{pc} / \mathrm{j}$ pour l'adulte et $0,022 \mu \mathrm{g} / \mathrm{kg} \mathrm{pc} / \mathrm{j}$ pour le jeune de moins de 14 ans (Leblanc et al., 2011) et sont comprises entre 8 et $16 \mu \mathrm{g} / \mathrm{j}$.

Nos résultats indiquent que les toxiques recherchés sont présents dans les matrices étudiées. Ce qui traduit la contamination des animaux. Cette contamination est probablement d'origine alimentaire car l'alimentation est la source principale de contamination des animaux, et les transferts des polluants vers ceux-ci s'effectuent par ingestion involontaire de sol par les ruminants au pâturage ou par les fourrages contaminés (Claire et Feindt, 2005). La présence de métaux dans l'alimentation du bétail, lorsqu'elle survient, entraîne principalement une accumulation dans les reins et les foies d'animaux (Royer et al., 2010). Par exemple, chez les animaux de rente, la teneur des abats en cadmium reflète parfaitement le cadmium que l'animal trouve dans son alimentation et son environnement (Burgat Sacaze et al., 1996). Cette source alimentaire a une double origine:

- Les plantes servant de nourriture (foins) cultivées sur des sols contaminés ou par l'environnement riche en ces métaux, peuvent accumuler ces métaux et contaminer les animaux nourris par ces plantes.

- Les farines animales utilisées pour nourrir le bétail sont fabriquées à bases de déchets de poissons eux-même contaminées depuis la chaîne alimentaire marine. Une fois ingérés, les polluants métalliques ont pour organes cibles les reins, le foie, les muscles et les os (Claire et Feix, 2005). Les métaux analysés sont inégalement repartis au niveau des organes matrices de notre étude. En effet,
l'ANOVA montre une différence significative entre les concentrations métalliques au niveau de ces organes. La disparité au niveau de la distribution tissulaire se justifie par les différences de fonctionnalité métabolique des organes et aussi par des origines des abats. En effet, certains contaminants du fait de leurs caractéristiques physicochimique et les phénomènes de spéciation s'accumuleraient plus facilement dans certains organes plutôt que d'autres (Kraemer et al., 2005). La bioaccumulation qui en découle, résulte du bilan entre d'une part, les entrées des métaux, via le processus d'adsorption et d'absorption aux travers des membranes biologiques à l'interface «organisme/milieu » et les mécanismes de séquestration dans les compartiments tissulaires et d'autre part les voies d'élimination et de décontamination (BOUDOU, 2004). On remarque que les échantillons de rognon sont les plus contaminés dans l'ensemble. Cette situation est due à la fonction éliminatrice du rognon, d'où la présence excessive des métaux dans cet organe.

Les origines peuvent aussi expliquer les disparités entre les concentrations, les viandes et abats importés provenant d'horizons divers, allant des Amériques à l'Asie en passant par l'Europe. Les différences fondamentales entre nos résultats et ceux de la littérature pourraient s'expliquer par les autres paramètres qui influencent la bioaccumulation chez l'animal tels que l'âge. Les différences au niveau du même organe entre les métaux sont plutôt liées aux caractéristiques physicochimiques des métaux.

L'alimentation reste la source majeure d'exposition aux métaux lourds de la population générale non professionnellement exposée et non fumeuse (Miquel, 2001; US EPA, 2003) et l'estimation de l'exposition humaine aux polluants métalliques repose sur les habitudes et les concentrations en éléments métalliques (Claire et al., 2005) à travers l'estimation des moyennes. Cependant, les différents types d'aliments ne contribuent pas à la même hauteur à cette exposition. Pour certains métaux, les sources sont bien identifiées comme le témoigne le Tableau 3. Certains aliments contribuent plus 
à l'exposition à un métal que d'autres. Cette contribution est liée à l'aptitude de l'aliment à concentrer le métal. Les légumes et les céréales sont les sources principales de cadmium, bien que le $\mathrm{Cd}$ soit également trouvé dans la viande mais à un degré moindre. Le cadmium s'accumule en particulier dans le rein et le foie, par conséquent les abats contiennent des concentrations relativement élevées. Le poisson contient seulement de petites quantités de cadmium alors que les crustacés et les mollusques peuvent accumuler de plus grandes quantités (Bliefert et Perraud, 2004). Les poissons sont les principales sources alimentaires du mercure quand les boissons, fruits et légumes accumulent le plomb. Parmi les sources d'origine animale, les viandes et abats sont concernés au niveau du plomb et du cadmium. Et aussi bien que les teneurs dans les produits carnés, soient quantitativement bien en dessous des limites réglementaires, les organes comestibles du porc contribuent à l'exposition totale chez l'homme (EFA, 2009, 2010).

Les produits carnés ne sont pas des vecteurs alimentaires de la contamination au mercure. En effet, seuls les poissons sont considérés comme les principales sources alimentaires de l'intoxication au mercure. L'exposition qui découle de notre étude ne présente pas de danger pour les consommateurs. Elle représente $0,2 \%$ de la DJT chez les adultes et $0,6 \%$ chez les enfants.

De façon générale, les expositions calculées sont supérieures aux estimations trouvées dans la bibliographie qui souvent indique que les consommateurs sont très peu nombreux à dépasser la DHTP.

En dehors du mercure, les viandes et abats sont des sources alimentaires potentielles du plomb et cadmium parce que susceptibles de concentrer les contaminants métaux lourds et métalloïdes des toxiques. L'exposition étant une fonction de la contamination (concentration) et de la consommation (quantité). Les doses d'exposition en cadmium et plomb via la consommation des viandes et abats importés apparaissent faibles chez les adultes et les enfants. Le quotient de danger est dans tous les cas très largement inférieur à 1 ; de l'ordre de $0,3 \%$ de la DJT du cadmium pour l'adulte et $1 \%$ de la DJT pour l'enfant. A pour le plomb, le quotient de danger est de l'ordre de $0,5 \%$ pour l'adulte et de $1 \%$ pour l'enfant.

Nos valeurs, notamment les doses journalières tolérables, restent nettement inférieures aux valeurs toxicologiques de référence et à celles rencontrées dans la littérature notamment en France. Mais en réalité, nos valeurs ne portent que sur l'exposition à ces toxiques apportés uniquement par les importations de viandes et d'abats de porc, bovins et ovins. Cette étude ne prend pas en compte toutes les autres sources potentielles de ces différents éléments. Surtout que pour certains de ces métaux, les sources principales ne sont pas les matrices étudiées. Notamment le mercure où aux valeurs actuelles de consommation, la dose d'exposition pour le jeune enfant représente $6 \%$ de la valeur toxicologique de référence. Ces résultats sont calculés sur la base des données de consommation nationales. Pour les grands consommateurs, ces données ne seraient pas réelles.

\section{Conclusion}

Ces données d'exposition montrent que la consommation des viandes et abats importés peut contribuer, pour certaines parties des animaux, (Rognon) au dépassement des valeurs de référence. Ces aliments analysés sont certes par rapport à certains éléments traces métalliques des sources d'exposition humaine à ces métaux, mais n'en sont pas principales. Le risque de surexposition de la population aux éléments métalliques toxiques par la consommation de viande et abats importés est avéré en raison de la contamination importante de certaines parties (Rognon) et de la contamination non négligeable d'autres sources comme les poissons pour le mercure et les fruits, légumes et eau pour le cadmium et plomb. Le risque apparaît plus grand si nous prenons en compte l'effet cumulatif au niveau de la toxicité des métaux. Néanmoins, il importe de conduire une étude générale prenant en compte non seulement les minéraux essentiels, oligoéléments et métaux traces métalliques toxiques, éléments organiques persistants, mais aussi l'ensemble des aliments, viandes, 
fruits et légumes, tubercules et féculents, céréales, alcools, boissons, etc., pour une évaluation complète de l'exposition alimentaire aux différents éléments. Cette mission impliquant tous les ministères (Agriculture, Santé, Ressources Animales, etc.) et confiée à une équipe pluridisciplinaire de toxicologues, nutritionnistes, vétérinaires, hygiénistes, etc.

\section{REFERENCES}

Boudou A. 2004. Mécanismes de bioaccumulation des métaux traces chez les organismes aquatiques, université bordeaux 1, place dr peyreau- 33120 arcachon. Journées : contamination décontamination des mollusques bivalves; ifremer6 nantes 26-27 mai 2004. livret des résumés.

AOAC. 2000. Officials Methods of Analysis: AOAC Official Method 971.21 Mercury in Foods, Flameless Atomic Absorption Spectrophotometric Method $\left(17^{\text {th }}\right.$ edn). AOAC: washington, DC.

Bliefert C, Perraud R. 2004. Chimie de l'environnement: air, eau, sols, déchets. Boeck université. pp. 373-374.

Boumehres A. 2010. Etude comparative des techniques d'extraction des éléments traces métalliques dans le foie, le rein et le lait et leur détermination par spectrophotométrie d'absorption atomique (flamme et four graphite).

Burgat-sacaze V, craste I, Guerre P. 1996. Le cadmium dans les chaînes alimentaires. Rev. Méd.Vét., 147(10): 671-680.

Commission Européenne. 2006: Règlement $\mathrm{CE} \mathrm{N}^{\circ} 1881 / 2006$ de la commission du 19 décembre 2006 (1881/2006) portant fixation de teneurs maximales pour certains contaminants dans les denrées alimentaires.

Claire L, Cyril F, François L. 2005. Contamination des Sols. Transfert s des Sols Vers les Animaux. Ed Sciences/ Ademe ; 216.

CSHPF (Conseil Supérieur d'Hygiène Publique de France, section des eaux). 1997. Ministère du travail et des affaires sociales- direction générale de la santé. Risques sanitaires liés aux boues d'épuration des eaux usées urbaines. edition lavoisier -tec et doc, paris 8ème $106 \mathrm{p}$.

Decloitre F., 1998: la part des différents aliments dans l'exposition au plomb, au cadmium et au mercure, en france. Cah. Nutr. Diét., 33(3): 167-175.

DGS. 1995. La diagonale des métaux, études sur la teneur en métaux de l'alimentation.

EFSA. 2009. Scientific opinion on cd in food. The Efsa Journal, 980: 1-139.

EFSA. 2010. Scientific opinion on lead in food. The Efsa Journal, 8(4): 1-147.

JEFCA. 2004. Joint expert committee mixt FAO/OMS food additives and contaminants: évaluation de certains additifs alimentaires et contaminants. Soixante et unième rapport du comité mixte FAO/OMS d'experts. Rome, OMS, June 2003.

JECFA. 2004. Evaluation of certain food additives and contaminants. 61th report of the joint FAO/WHO expert committee on food additives and contaminants. WHO Geneva.

JECFA, 2011. Evaluation of certain food additives and contaminants $72^{\text {nd }}$ report of the joint FAO/WHO expert committee on food additive who technical report series 959.

Kraemer LD, Campbell PGC, Hare L. 2005. Dynamics of $\mathrm{Cd}, \mathrm{Cu}$ and $\mathrm{Zn}$ accumulation in organs and sub-cellular fraction in field transplanted juvenile yellow perch (perca flavescens). Environment Pollution, 138: 324-337.

Leblanc J-C, Yoon H, Kombadjian A, Verger P. 2000. Nutritional intakes of vegetarian

populations in france, European Journal of Clinical Nutrition, 54: 1-7.

Leblanc JC, Guérin T, Verger P, Volatier JL. 2004. Etude de l'alimentation totale française. Mycotoxines, minéraux et éléments traces. INRA, Ministère de l'Agriculture, de l'Alimentation, de la Pêche et des Affaires Rurales, 72 p.

Leblanc J-C, Guerin T, Noël L, CalamassiTran G, Volatier J-L, Verger P. 2005. Dietary exposure estimates of 18 elements from the 1 st french total diet study. Food Addit. Contam., 22(7): 624641. 
Leblanc JC, Sirot V, Volatier JL, Aouachria NB. 2011. Etude des Consommations Alimentaires de produits de la mer et Imprégnations aux éléments traces, Polluants et Oméga 3 (CALIPSO). Afssa, Ministère de l'Agriculture et de la Pêche, INRA.

Noël L, Leblanc JC, Guérin T. 200. Determination of several elements in duplicate meals from catering stablishment using closed vessel microwave digestion with inductively coupled plasma mass spectrometry detection: estimation of daily dietary intake. Food Additives and Contaminants, 20(1): 44-56.
Royer E, Lebas N. 2010. Effet de la présence de cd et du pb dans l'alimentation du porc sur les teneurs dans les tissus comestibles. $13^{\text {ème }}$ jsmtv. www.itp.asso.fr/ ouverture pdf file=jsmtv 2010royer.pdf.

Picot A. 2003. Intoxication de l'organisme par les métaux lourds et autres toxiques. Le mercure, le plomb et le cadmium, trois métaux traces toxiques. Conférence ADNO, 2003 Paris, 29 nov 2003.

Sétra. 2004. Services d'études techniques des routes et autoroutes, note d'information, déc 2004. Economie, Environnement, Conception 73. 